\title{
OPTIMAL DECAY RATE FOR DEGENERATE PARABOLIC EQUATIONS ON NONCOMPACT MANIFOLDS
}

\author{
DANIELE ANDREUCCI \\ DIPARTIMENTO DI SCIENZE DI BASE E APPLICATE PER L'INGEGNERIA, SAPIENZA \\ UNIVERSITÀ DI ROMA \\ VIA A.SCARPA 16, 00161 ROMA, ITALY \\ ANATOLI F. TEDEEV \\ SOUTH MATHEMATICAL INSTITUTE OF VSC RAS, VLADIKAVKAZ \\ 362027, VLADIKAVKAZ, MARKUSA, 22 RUSSIA
}

\begin{abstract}
We consider an initial value problem for a doubly degenerate parabolic equation in a noncompact Riemannian manifold. The geometrical features of the manifold are coded in either a Faber-Krahn inequality or a relative Faber-Krahn inequality. We prove optimal decay and space-time local estimates of solutions. We employ a simplified version of the by now classical local approach by DeGiorgi, Ladyzhenskaya-Uraltseva, DiBenedetto which is of independent interest even in the euclidean case.

This is an Accepted Manuscript version of the following article, accepted for publication in Methods Appl. Anal. 22 (2015), no. 4, 359-376. The Pub-

lishers retain all rights. The arcticle is available online at https://dx.doi.org/10.4310/MAA.2015.v22.n4.a2
\end{abstract}

\section{INTRODUCTION.}

Let $(M, g)$ be a smooth Riemannian manifold of dimension $N \geq 2$. We assume $M$ to be connected, complete and noncompact. More precise assumptions on the metric of $M$ will be given below.

We look at nonnegative solutions of the equations of the type of

$$
\begin{aligned}
u_{t} & =\operatorname{div}\left(u^{m-1}|\nabla u|^{p-2} \nabla u\right), & & \text { in } M \times(0, T), \\
u(x, 0) & =u_{0}(x), & & x \in M .
\end{aligned}
$$

The operators div and $\nabla$ are of course understood in the sense of the Riemannian metric. We always assume $p>1, m>0, m+p>3$. This range of parameters is often referred to as the slow diffusion case.

We prove optimal $L^{1}-L^{\infty}$ a priori estimates. In the linear case $m=1, p=2$ such bounds are well known, see [13], [17], [18], [11]. In the degenerate case when $m=1$ we quote [12], [7], [15], [16]. We also quote some papers whose methods, even when stated in Euclidean spaces, are amenable to application to manifolds, being based on embedding estimates known to extend to this setting under some geometrical assumptions: [6], [8], [21], [22].

Key words and phrases. doubly degenerate parabolic equation, noncompact Riemannian manifold, relative Faber-Krahn inequality, optimal global and local bounds.

AMS Classification: 35K55, 35K65, 35B40.

The first author is member of the Gruppo Nazionale per la Fisica Matematica (GNFM) of the Istituto Nazionale di Alta Matematica (INdAM). The second author was supported by Sapienza University of Rome funding for Visiting Professors.

E-mail: daniele.andreucci@sbai.uniroma1.it; a_tedeev@yahoo.com. 
In order to describe our results we need introduce some notation; we let for $R>0$

$$
B_{R}\left(x_{0}\right)=\left\{x \in M \mid \operatorname{dist}\left(x, x_{0}\right)<R\right\}, \quad V(R)=\left|B_{R}\left(x_{0}\right)\right|,
$$

where $x_{0} \in M$ is a fixed point; in the following we'll often drop the dependence on $x_{0}$ from the notation. We also define the two functions

$$
\begin{gathered}
\mathcal{R}:(0, \infty) \rightarrow(0, \infty) \text { inverse function to } s \mapsto V(s) ; \\
\mathcal{Z}:(0, \infty) \rightarrow(0, \infty) \text { inverse function to } s \mapsto s^{p} V(s)^{p+m-3} .
\end{gathered}
$$

It is known that solutions to (1.1) in a domain $\Omega \subset \boldsymbol{R}^{N}$ with noncompact boundary, with zero boundary data of Neumann type satisfy decay estimates of the form

$$
\|u(t)\|_{\infty, \Omega} \sim \frac{\left\|u_{0}\right\|_{1, \Omega}}{V\left(\mathcal{Z}\left(t\left\|u_{0}\right\|_{1, \Omega}^{m+p-3}\right)\right)}, \quad t \gg 1,
$$

where we extend our definitions above to the case $M=\Omega$. Indeed (1.3) was proven in [3] for expanding domains, and in [2], [4] for narrowing domains, in the case of (1.1). In the linear case the estimate (1.3) was proven by Gushchin (see [24] and the references therein) in $\boldsymbol{R}^{N}$, and in [17], [18] in Riemannian manifolds.

Our first aim in this paper is to obtain the analog of (1.3) in Riemannian manifolds. In the Riemannian setting we discriminate between manifolds satisfying a global or a relative Faber-Krahn inequality. This distinction replaces the one between expanding and narrowing subdomains of $\boldsymbol{R}^{N}$.

Definition 1.1. $(M, g)$ satisfies a global Faber-Krahn inequality for a given $p>1$ and a function $\Lambda_{p}: \boldsymbol{R}^{+} \rightarrow \boldsymbol{R}^{+}$if for any $v>0$ and precompact domain $\Omega \subset M$ with $|\Omega|=v$ we have

$$
\Lambda_{p}(v) \int_{\Omega}|\varphi|^{p} \mathrm{~d} \mu \leq \int_{\Omega}|\nabla \varphi|^{p} \mathrm{~d} \mu
$$

for all $\varphi \in W_{0}^{1, p}(\Omega)$.

Remark 1.2. It is a classical fact that when $p=2$ the optimal choice of the constant on the left hand side of (1.4) is given by the first eigenvalue $\lambda_{1}(\Omega)$ of the Laplacian; a similar remark holds true for the case $p \neq 2$, see [27]. The classical Faber-Krahn inequality, when $p=2$, and its counterparts for $p \neq 2$ essentially consist in a bound from below for such optimal constants given in terms of the volume of $\Omega$; we refer the reader to [28].

We also need the following assumption:

H.1 There exists an $\alpha>0$ such that the function $s \mapsto s^{-\alpha} \Lambda_{p}\left(s^{-1}\right), s>0$, is nondecreasing, and $s \mapsto \Lambda_{p}(s), s>0$, is decreasing.

Theorem 1.3. Assume that $M$ satisfies a global Faber-Krahn inequality, with $\Lambda_{p}$ as in H.1. Let $u_{0} \in L^{1}(M)$ be nonnegative. Then problem (1.1), (1.2) has a solution defined in $M \times(0, \infty)$, and for all $t>0$

$$
\|u(t)\|_{\infty, M}^{m+p-3} \Lambda_{p}\left(\frac{\left\|u_{0}\right\|_{1, M}}{\|u(t)\|_{\infty, M}}\right) \leq \gamma t^{-1} .
$$

In Theorem 1.3 and in the rest of the paper we denote by $\gamma, \gamma_{1}, \ldots$, possibly different positive constants depending only on the parameters of the problem $p, m$, $N, \ldots$, and on the constants $\alpha, \alpha_{j}$ appearing in the assumptions. We also assume that volume is normalized so that $V(1)=1$. 
Remark 1.4. One can check easily, from the definition of the function $\mathcal{Z}$, that (1.5) implies its possibly more transparent version

$$
\|u(t)\|_{\infty, M} \leq \gamma \frac{\left\|u_{0}\right\|_{1, M}}{V\left(\mathcal{Z}\left(t\left\|u_{0}\right\|_{1, M}^{m+p-3}\right)\right)}, \quad t>1,
$$

provided we have for some $\alpha_{0}>0$

$$
\Lambda_{p}(v) \geq \frac{\alpha_{0}}{\mathcal{R}(v)^{p}}, \quad v>1,
$$

which is known to hold true in many important examples. See however Section 1.1 below for further comments and a counterexample.

Remark 1.5. It is well known that if a suitable isoperimetric inequality is available in $M$, then the Faber-Krahn inequality holds true, see e.g, [14]. One can apply the symmetrization technique to get such bound; see [10], [9]. On this matter we also quote [19]. In [14] also the relation between Sobolev type embeddings and Faber-Krahn inequality is discussed; actually the two are equivalent at least if $\Lambda_{p}$ is the same as in the Euclidean space.

For a nonnegative locally integrable function $f$ and $\bar{R} \geq 1$ define

$$
\|f\|_{\bar{R}}=\sup _{R \geq \bar{R}} R^{-\frac{p}{p+m-3}} V(R)^{-1} \int_{B_{R}} f \mathrm{~d} \mu .
$$

Our local in time existence results can be stated in terms of the norm just defined.

Theorem 1.6. Assume that $M$ satisfies a global Faber-Krahn inequality, with $\Lambda_{p}$ as in H.1, and in (1.7). Let $u_{0} \in L_{\text {loc }}^{1}(M)$ be nonnegative, and such that $\left\|u_{0}\right\|_{\bar{R}}<$ $\infty$. Then problem (1.1), (1.2) has a solution defined in $M \times\left(0, T_{\bar{R}}\right)$, where $T_{\bar{R}}=$ $\gamma_{0}\left\|u_{0}\right\| \|_{\bar{R}}^{3-p-m}$, and such that for $0<t<T_{\bar{R}}, R \geq \bar{R}$,

$$
\begin{gathered}
\|u(t)\|_{\bar{R}} \leq \gamma\left\|u_{0}\right\|_{\bar{R}}, \\
\left\|u_{0}\right\|_{\infty, B_{R}} \leq \gamma \frac{V(R) R^{\frac{p}{p+m-3}}\left\|u_{0}\right\|_{\bar{R}}}{V\left(\mathcal{Z}\left(t V(R)^{p+m-3} R^{p}\left\|u_{0}\right\| \frac{p+m-3}{R}\right)\right)} .
\end{gathered}
$$

Remark 1.7. It is worth mentioning that when $\left\|u_{0}\right\|_{\bar{R}} \rightarrow 0$ as $\bar{R} \rightarrow+\infty$, Theorem 1.6 actually implies existence of a solution defined for all $t>0$. Indeed, since $T_{\bar{R}} \rightarrow+\infty$ as $\bar{R} \rightarrow+\infty$ in the case at hand, we immediately derive from (1.9) a priori sup estimates in any compact of $M \times(0,+\infty)$. This remark and a standard approximation procedure e.g., by solutions with smooth compactly supported initial data yield the result.

1.1. Examples. Let $M_{k}=\boldsymbol{R}^{k} \times S^{N-k}$. Here $1 \leq k<N$ and $S^{N-k}$ is the $N-k$ dimensional sphere. In this case (see [14])

$$
\Lambda_{p}(v)=\gamma(N, k) \min \left(v^{-\frac{p}{N}}, v^{-\frac{p}{k}}\right),
$$

and (1.5) amounts to

$$
\begin{aligned}
& \|u(t)\|_{\infty, M_{k}} \leq \gamma \\
& \quad \times \max \left(t^{-\frac{k}{k(m+p-3)+p}}\left\|u_{0}\right\|_{1, M_{k}}^{\frac{p}{k(m+p)+p}}, t^{-\frac{N}{N(m+p-3)+p}}\left\|u_{0}\right\|_{1, M_{k}}^{\frac{p}{N(m+p-3)+p}}\right) .
\end{aligned}
$$

More generally, the function $\Lambda_{p}$ for product manifolds is given in [14] as a suitable combination of the corresponding functions of the factors. 
This example can be used to show that our upper estimate, that is (1.11) in this case, is actually optimal. Indeed, the Cauchy problem (1.1)-(1.2) set in $\boldsymbol{R}^{k}$ has a well known self-similar solution globally integrable in space, exhibiting the asymptotic decay rate given by the first term in the max function in (1.11). However, this solution is clearly also a solution of the Cauchy problem in $M_{k}$, since it does not depend on the last $N-k$ variables, and it is globally integrable in $M_{k}$, since the factor $S^{N-k}$ is compact. Hence the claim.

As a further example we consider the revolution surface

$$
M^{\alpha}=\left\{x=\left(x^{\prime}, x_{N+1}\right)|| x^{\prime} \mid=x_{N+1}^{\alpha}\right\} \subset \boldsymbol{R}^{N+1},
$$

with $0<\alpha<1$. Invoking the connection between the isoperimetric and FaberKrahn inequalities, see Remark 1.5, we can check by fairly explicit calculations that in this case the function $\Lambda_{p}$ can be written as in (1.10), when we formally replace $k$ there with $\alpha(N-1)+1$.

Remark 1.8. The linear case $m=1, p=2$ of the heat equation has been intensively studied. For example in [20] Section 1.2, the authors consider a class of manifolds falling within the scope of our results: specifically manifolds $M_{k} \# M_{n}, 2<k<n<$ $N$ where $M_{k}, M_{n}$ are as above and the symbol \# denotes connection by a compact handle. In this case we can take $\Lambda_{p}$ as in (1.10), whence our estimate (1.11) follows. As a further argument supporting its optimality we notice that the authors of [20] prove two sided estimates of the heat kernel reducing to the same decay rate we obtain in (1.11) when we formally let $m=1, p=2$ there. However our approach is completely different from the one in [20].

As a side remark we note that $M_{k} \# M_{n}$ provides a counterexample where (1.7) is not fulfilled. Indeed for large $R, V(R) \simeq R^{n}$.

\subsection{The case of relative Faber-Krahn inequalities.}

Definition 1.9. $(M, g)$ satisfies a relative Faber-Krahn inequality for a given $p>1$ and a function $\Lambda_{p}: \boldsymbol{R}^{+} \times \boldsymbol{R}^{+} \rightarrow \boldsymbol{R}^{+}$if for any $v>0, R>1$, and precompact domain $\Omega \subset B_{R}\left(x_{0}\right)$ with $|\Omega|=v \leq\left|B_{R}\left(x_{0}\right)\right| / 2$ we have

$$
\Lambda_{p}(v, R) \int_{\Omega}|\varphi|^{p} \mathrm{~d} \mu \leq \int_{\Omega}|\nabla \varphi|^{p} \mathrm{~d} \mu,
$$

for all $\varphi \in W_{0}^{1, p}(\Omega)$.

We need the following assumptions:

H.2 There exist $\alpha_{1}, \alpha_{2} \in(0,1)$ such that both functions $V(s) / s^{\alpha_{1}}, s^{\alpha_{2}} / V(s)$

are nondecreasing for $s \geq 1$.

H.3 The function $\Lambda_{p}$ takes the form

$$
\Lambda_{p}(v, R)=\gamma \min \left(\frac{V(R)^{p}}{v^{p} R^{p}}, v^{-\frac{p}{N}}\right), \quad v>0, R>1 .
$$

Assumption H.2 immediately implies that for all $c \geq 1, R \geq 1$

$$
c^{-\alpha_{2}} V(c R) \leq V(R) \leq c^{-\alpha_{1}} V(c R) .
$$

Remark 1.10. The form of $\Lambda_{p}$ in H.3 is natural, at least in the case when $M$ is a narrowing domain in $\boldsymbol{R}^{N}$, since in that case the ratio $V(R) / R$ takes the intuitive meaning of area of $B_{R}(0) \cap M$ (see [23], [4]). In the case of Riemannian manifolds a similar form of $\Lambda_{p}$ has been used in [17]. 
The following definition is instrumental in our approach:

$$
F(s)=\frac{s}{V(s)}, \quad s>1 ; \quad F(s)=\frac{1}{V(1)}=1, \quad 0 \leq s \leq 1 .
$$

As a consequence of H.2, $F$ is non-decreasing and

$$
F^{\prime}(s) \leq \frac{1}{V(s)}, \quad s>0 .
$$

Let us define the standard Barenblatt exponent

$$
\beta=N(p+m-3)+p .
$$

Theorem 1.11. Assume that $M$ satisfies a relative Faber-Krahn inequality with $\Lambda_{p}$ as in (1.13) and in H.2, H.3. Let $u_{0} \in L_{\mathrm{loc}}^{1}(M)$ be nonnegative, and such that $\left\|u_{0}\right\|_{\bar{R}}<\infty$. Then problem (1.1), (1.2) has a solution defined in $M \times\left(0, T_{0}\right)$, where $T_{0}=\gamma_{0}\left\|u_{0}\right\|_{\frac{3}{R}}^{3-p-m}$, and such that for $0<t<T_{0}, R \geq \bar{R}$,

$$
\begin{gathered}
\|u(t)\|_{\bar{R}} \leq \gamma\left\|u_{0}\right\|_{\bar{R}}, \\
\left\|u_{0}\right\|_{\infty, B_{R}} \leq \gamma R^{\frac{p}{p+m-3}} \max \left\{t^{-\frac{N}{\beta}}\left\|u_{0}\right\| \frac{p / \beta}{R}, t^{-\frac{1}{2 p+m-3}}\left\|u_{0}\right\| \frac{p}{\bar{R}+m-3}\right\} .
\end{gathered}
$$

Moreover, if $\left\|u_{0}\right\|_{1}<\infty$, then for any $R>1$ and any $t>0$

$$
\|u(t)\|_{\infty, B_{R}} \leq \gamma \max \left\{t^{-\frac{N}{\beta}}\left\|u_{0}\right\|_{1}^{\frac{p}{\beta}}, t^{-\frac{1}{2 p+m-3}}\left\|u_{0}\right\|_{1}^{\frac{p}{2 p+m-3}} F(R)^{\frac{p}{2 p+m-3}}\right\} .
$$

The global boundedness of $u(t)$ is connected to the moment

$$
I(t)=\int_{M} u(x, t) F\left(\operatorname{dist}\left(x, x_{0}\right)\right) \mathrm{d} \mu, \quad I_{0}=I(0) .
$$

Theorem 1.12. Assume that $M$ satisfies a relative Faber-Krahn inequality with $\Lambda_{p}$ as in (1.13) and in H.2, H.3. Let $u_{0} \in L^{1}(M)$ be such that $I_{0}<\infty$. Then problem (1.1), (1.2) has a solution defined in $M \times(0,+\infty)$ and for all $t>0$ we have

$$
\|u(t)\|_{\infty, M} \leq \gamma \mathcal{U}(t),
$$

where

$$
\begin{aligned}
& \mathcal{U}(t)=\max \left\{t^{-\frac{N}{\beta}}\left\|u_{0}\right\|_{1, M}^{\frac{p}{\beta}}, t^{-\frac{1}{2 p+m-3}} I_{0}^{\frac{p}{2 p+m-3}},\right. \\
&\left.t^{-\frac{1}{2 p+m-3}}\left\|u_{0}\right\|_{1, M}^{\frac{p}{2 p+m-3}} F\left[\mathcal{Z}\left(\left\|u_{0}\right\|_{1, M}^{m+p-3} t\right)\right]^{\frac{p}{2 p+m-3}}\right\} .
\end{aligned}
$$

Therefore for large enough $t$ (1.5) still holds true.

Remark 1.13. We need in Theorem 1.12 the assumption $I_{0}<\infty$, which is known to be necessary to obtain uniform sup estimates in narrowing domains, see [26], [2]. However the form of the sup estimate for large times is the same in Theorem 1.12 as in (1.6). Indeed for large $t$ the greatest of the three arguments in the max function in (1.20) is the third one. On substituting in it the definition of $F$ and then using the definition of $\mathcal{Z}$ we see that this argument equals the right hand side of (1.6). 


\subsection{Example of $M$ with relative Faber-Krahn inequality. Let}

$$
M=\left\{x=\left(x^{\prime}, x_{N+1}\right)|| x^{\prime} \mid=f\left(x_{N+1}\right)\right\} \subset \boldsymbol{R}^{N+1}
$$

be the surface in $\boldsymbol{R}^{N+1}$ obtained by revolution of the graph of $f$, where $f \in C^{\infty}(\boldsymbol{R})$ is a positive function such that $f(s)=|s|^{-b}$ for $|s|>1$, with $0<b<1 / N$. In this case calculations similar to the ones in [4] yield

$$
V(s)=\gamma s^{1-N b}(1+\mathrm{o}(1)), \quad s \rightarrow \infty .
$$

Thus (1.19) implies

$$
\begin{array}{ll}
\|u(t)\|_{\infty, M} \leq \gamma\left\|u_{0}\right\|_{1, M}^{\frac{p}{\beta_{M}}} t^{-\frac{1-N b}{\beta_{M}}}, & t \gg 1, \\
\|u(t)\|_{\infty, M} \leq \gamma\left\|u_{0}\right\|_{1, M}^{\frac{p}{\beta}} t^{-\frac{N}{\beta}}, & t \ll 1,
\end{array}
$$

where $\beta=N(p+m-3)+p, \beta_{M}=(1-N b)(m+p-3)+p$.

Theorem 1.3 is proven in Section 2. The proof of Theorem 1.6 follows from very similar arguments, combined with a suitable localization procedure (see also Section 4), and is therefore omitted.

The proof of Theorem 1.11 is prepared by an embedding result given in Section 3, and then presented in Section 4. Finally, Theorem 1.12 is proven in Section 5.

Actually, for the sake of brevity we confine ourselves to proving the sup bounds contained in the Theorems above, assuming we are dealing, for example, with solutions to Dirichlet problems, with vanishing boundary data, in some ball $B_{j}$. The existence statement, and the sup bounds as well of course, are then recovered as $j \rightarrow \infty$ by a standard approximation argument relying on by now classical compactness results for equibounded solutions.

Acknowledgments. We are indebted to the referee for several useful remarks.

\section{Proof of Theorem 1.3.}

Let $f \in W^{1, p}(M)$ be compactly supported and $k \geq 0$; then it follows from applying the Faber-Krahn inequality to $\varphi=(f-k)_{+}$that

$$
\int_{M}(f-k)_{+}^{p} \mathrm{~d} \mu \leq \Lambda_{p}(|\{f>k\}|)^{-1} \int_{M}\left|\nabla(f-k)_{+}\right|^{p} \mathrm{~d} \mu .
$$

We also infer by means of Hölder inequality that for $0<q<p$

$$
\begin{aligned}
\int_{M}(f-k)_{+}^{q} \mathrm{~d} \mu \leq|\{f>k\}|^{1-\frac{q}{p}} \Lambda_{p}(|\{f>k\}|)^{-\frac{q}{p}} & \\
& \times\left(\int_{M}\left|\nabla(f-k)_{+}\right|^{p} \mathrm{~d} \mu\right)^{\frac{q}{p}} .
\end{aligned}
$$

In the following we denote by $u$ a compactly supported (for all times) solution to (1.1)-(1.2), or a solution to a Dirichlet problem set in a ball $B_{j}$, with vanishing boundary data, in the spirit of a procedure of approximation as remarked in the Introduction. We need the auxiliary result 
Lemma 2.1. Let $\vartheta>0$, and $\vartheta>2-m$ if $m<1$, be fixed, and define $s=$ $(p+m+\vartheta-2) / p$. Fix also $a_{1}>a_{2}>0, \tau_{1}>\tau_{2}>0, R_{2}>R_{1}$. Then

$$
\begin{aligned}
& \sup _{\tau_{1}<\tau<t} \int_{B_{R_{1}}}\left(u(\tau)-a_{1}\right)_{+}^{\vartheta+1} \mathrm{~d} x+\int_{\tau_{1}}^{t} \int_{B_{R_{1}}}\left|\nabla\left(u-a_{1}\right)_{+}^{s}\right|^{p} \mathrm{~d} x \mathrm{~d} \tau \leq \gamma\left(\frac{a_{1}}{a_{1}-a_{2}}\right)^{|m-1|} \\
& \times\left\{\left(\tau_{1}-\tau_{2}\right)^{-1} \int_{\tau_{2}}^{t} \int_{B_{R_{2}}}\left(u-a_{2}\right)_{+}^{\vartheta+1} \mathrm{~d} x \mathrm{~d} \tau+\left(R_{2}-R_{1}\right)^{-p} \int_{\tau_{2}}^{t} \int_{B_{R_{2}}}\left(u-a_{2}\right)_{+}^{s p} \mathrm{~d} x \mathrm{~d} \tau\right\} .
\end{aligned}
$$

The last integral in (2.3) can be dropped from the right hand side, provided we formally replace on both sides $B_{R_{1}}$ and $B_{R_{2}}$ with $M$. We refer to this version of the estimate as to the global version of (2.3).

We omit the routine proof of Lemma 2.1, which is essentially obtained by multiplying (1.1) by $(u-k)_{+}^{\vartheta} \zeta^{p}$, where $\zeta$ is a suitable cutoff function, and then integrating by parts; see e.g., [5].

Proof of Theorem 1.3. Let us define for $h_{0}>h_{\infty}>0, \tau_{0}>\tau_{\infty}>0$, and $i=0,1$, $2, \ldots$,

$$
k_{i}=h_{\infty}+\left(h_{0}-h_{\infty}\right) 2^{-i}, \quad t_{i}=\tau_{\infty}+\left(\tau_{0}-\tau_{\infty}\right) 2^{-i}, \quad f_{i}=\left(u-k_{i}\right)_{+}^{\frac{p+m+\vartheta-2}{p}} .
$$

Next we infer from (2.2) and Young's inequality, for any fixed time $\tau$, and $\sigma>0$, $0<q<p$ to be chosen,

$$
\begin{aligned}
\int_{M} f_{i+1}^{q} \mathrm{~d} \mu & \leq \Lambda_{p}\left(\left|\left\{u>k_{i+1}\right\}\right|\right)^{-\frac{q}{p}}\left|\left\{u>k_{i+1}\right\}\right|^{1-\frac{q}{p}}\left(\int_{M}\left|\nabla f_{i+1}\right|^{p} \mathrm{~d} \mu\right)^{\frac{q}{p}} \\
& \leq \sigma^{\frac{p}{q}} \int_{M}\left|\nabla f_{i+1}\right|^{p} \mathrm{~d} \mu+\gamma \sigma^{-\frac{p}{p-q}} \Lambda_{p}\left(\left|\left\{u>k_{i+1}\right\}\right|\right)^{-\frac{q}{p-q}}\left|\left\{u>k_{i+1}\right\}\right| .
\end{aligned}
$$

On integrating this inequality in time, we obtain

$$
\begin{aligned}
& \int_{t_{i+1}}^{t} \int_{M} f_{i+1}^{q} \mathrm{~d} \mu \mathrm{d} \tau \leq \sigma^{q} \int_{t_{i+1}}^{t} \int_{M}\left|\nabla f_{i+1}\right|^{p} \mathrm{~d} \mu \\
& \quad+\gamma t \sigma^{-\frac{p}{p-q}} \Lambda_{p}\left(\sup _{t_{i+1}<\tau<t}\left|\left\{u>k_{i+1}\right\}\right|\right)^{-\frac{q}{p-q}} \sup _{t_{i+1}<\tau<t}\left|\left\{u>k_{i+1}\right\}\right| .
\end{aligned}
$$

Select from now on

$$
q=\frac{p(1+\vartheta)}{p+m-2+\vartheta} .
$$


On combining now (2.4) and the global version of (2.3), where we let $a_{1}=k_{i}$, $a_{2}=k_{i+1}, \tau_{1}=t_{i}, \tau_{2}=t_{i+1}$, we get for a suitable choice of $\sigma>0$

$$
\begin{aligned}
\sup _{t_{i}<\tau<t} \int_{M} f_{i}^{q} \mathrm{~d} \mu+ & \int_{t_{i}}^{t} \int_{M}\left|\nabla f_{i}\right|^{p} \mathrm{~d} \mu \mathrm{d} \tau \leq \varepsilon \int_{t_{i+1}}^{t} \int_{M}\left|\nabla f_{i+1}\right|^{p} \mathrm{~d} \mu \\
+\gamma \gamma_{1}^{i} \varepsilon^{-\frac{q}{p-q}} t\left(\tau_{0}-\tau_{\infty}\right)^{-\frac{p}{p-q}}\left(\frac{h_{0}}{h_{0}-h_{\infty}}\right)^{\frac{p|m-1|}{p-q}} & \\
& \times \Lambda_{p}\left(\sup _{t_{i+1}<\tau<t}\left|\left\{u>k_{i+1}\right\}\right|\right)^{-\frac{q}{p-q}} \sup _{t_{i+1}<\tau<t}\left|\left\{u>k_{i+1}\right\}\right|,
\end{aligned}
$$

whence, by suitably selecting $\varepsilon>0$ and by iterating on $i$, we get

$$
\begin{aligned}
& \sup _{\tau_{0}<\tau<t} \int_{M}\left(u(\tau)-h_{0}\right)_{+}^{1+\vartheta} \mathrm{d} x \leq \gamma t\left(\tau_{0}-\tau_{\infty}\right)^{-\frac{p}{p-q}}\left(\frac{h_{0}}{h_{0}-h_{\infty}}\right)^{\frac{p|m-1|}{p-q}} \\
& \times \Lambda_{p}\left(\sup _{\tau_{\infty}<\tau<t}\left|\left\{u>h_{\infty}\right\}\right|\right)^{-\frac{q}{p-q}} \sup _{\tau_{\infty}<\tau<t}\left|\left\{u>h_{\infty}\right\}\right| .
\end{aligned}
$$

We complete the proof by means of a second iterative process; to this end we introduce for $k>0$ and $n=0,1,2, \ldots$ the sequences

$$
K_{n}=k\left(1-2^{-n-1}\right), \quad \bar{K}_{n}=\left(K_{n}+K_{n+1}\right) / 2, \quad t_{n}^{\prime}=t\left(1-2^{-n-1}\right),
$$

Then we apply (2.5) with $\tau_{0}=t_{n+1}^{\prime}, \tau_{\infty}=t_{n}^{\prime}, h_{0}=\bar{K}_{n}, h_{\infty}=K_{n}$ to conclude

$$
\begin{array}{r}
Y_{n+1}:=\sup _{t_{n+1}^{\prime}<\tau<t}\left|\left\{u>K_{n+1}\right\}\right| \leq \gamma \gamma_{1}^{n} k^{-(1+\vartheta)} \sup _{t_{n+1}^{\prime}<\tau<t} \int_{M}\left(u-\bar{K}_{n}\right)_{+}^{1+\vartheta} \mathrm{d} x \\
\leq \gamma \gamma_{1}^{n} t^{-\frac{q}{p-q}} k^{-(1+\vartheta)} \Lambda_{p}\left(Y_{n}\right)^{-\frac{q}{p-q}} Y_{n} .
\end{array}
$$

It follows from Lemma 5.6 p.65 of [25], and from assumption H.1, that $Y_{n} \rightarrow 0$ as $n \rightarrow \infty$ and therefore $\|u(t)\|_{\infty} \leq k$, provided

$$
t^{-\frac{q}{p-q}} k^{-(1+\vartheta)} \Lambda_{p}\left(Y_{0}\right)^{-\frac{q}{p-q}} \leq \delta,
$$

for a suitable $\delta=\delta(m, p, q)>0$. Next we note that

$$
Y_{0} \leq 2 k^{-1}\left\|u_{0}\right\|_{1} .
$$

Therefore, (2.6) is satisfied if

$$
t^{-1} k^{-(p+m-3)} \Lambda_{p}\left(k^{-1}\left\|u_{0}\right\|_{1}\right)^{-1} \leq 2^{-\alpha} \delta^{\frac{p+m-3}{1+\vartheta}} .
$$

Finally we choose $k$ as

$$
\left(k\left\|u_{0}\right\|_{1}^{-1}\right)^{p+m-3} \Lambda_{p}\left(k^{-1}\left\|u_{0}\right\|_{1}\right)=2^{1+\alpha} \delta^{-\frac{p+m-3}{1+\vartheta}} t^{-1}\left\|u_{0}\right\|_{1}^{-(p+m-3)} .
$$

Then (2.7) holds true and since $\|u(t)\|_{\infty} \leq k$ the sought after result readily follows. 


\section{A multiplicative inequality implied By the Relative Faber-Krahn} INEQUALITY.

Define for all $\theta>0,0<c<q, R>0$, and a sufficiently regular $f \geq 0$,

$$
\begin{aligned}
& E_{\theta}=\int_{B_{R}} f^{\theta} \mathrm{d} \mu, \quad E=E_{c}^{\frac{q}{q-c}} E_{q}^{-\frac{c}{q-c}}, \\
& \omega(E, R)=\max \left\{1, R V(R)^{-1} E^{\frac{N-1}{N}}\right\} .
\end{aligned}
$$

Lemma 3.1. Assume that $M$ satisfies a relative Faber-Krahn inequality with $\Lambda_{p}$ as in (1.13), H.3. Assume that $f$ is a Sobolev function such that $\nabla f \in L^{p}(M)$, and $\operatorname{supp} f \subset B_{R}$. Then there exists a constant $\vartheta_{0}(q)>0$ such that if

$$
E \leq \vartheta_{0} V(R),
$$

then for all $1 \leq q \leq p, 0<c<q$,

$$
\|f\|_{q, B_{R}} \leq \gamma \omega(E, R) E^{\frac{1}{q}-\frac{1}{p}+\frac{1}{N}}\|\nabla f\|_{p, B_{R}} .
$$

Remark 3.2. The estimate (3.2) is in fact true for $q(N-p) \leq N p$. However, here we use it only for $q \leq p$ (the proof for $q>p$ may be given following [4]). In this connection we remark that Hölder's inequality yields

$$
E \leq|\operatorname{supp} f| \text {. }
$$

Proof. Let $A(\lambda)=\left\{x \in B_{R}: f(x)>\lambda\right\}$; then we have for any $k>0$

$$
\begin{aligned}
E_{q}=\int_{A(k)}(f-k+k)^{q} \mathrm{~d} \mu & +\int_{B_{R} \backslash A(k)} f^{q} \mathrm{~d} \mu \\
& \leq 2^{q-1} \int_{A(k)}(f-k)^{q} \mathrm{~d} \mu+2^{q-1} k^{q}|A(k)|+k^{q-c} E_{c} .
\end{aligned}
$$

Observing that $|A(k)| \leq k^{-c} E_{c}$, we get from (3.4)

$$
E_{q} \leq 2^{q-1} \int_{A(k)}(f-k)^{q} \mathrm{~d} \mu+\left(2^{q-1}+1\right) k^{q-c} E_{c} .
$$

Select now $k$ as follows

$$
\left(2^{q-1}+1\right) k^{q-c} E_{c}=E_{q} / 2
$$

that is

$$
k=\left(\frac{E_{q}}{E_{c}} \frac{1}{2^{q}+2}\right)^{1 /(q-c)} .
$$

From (3.5) and (3.6) it follows that

$$
E_{q} \leq 2^{q} \int_{A(k)}(f-k)^{q} \mathrm{~d} \mu .
$$


We bound the right hand side of (3.7) by means of Hölder's inequality as

$$
\begin{aligned}
& \int_{A(k)}(f-k)^{q} \mathrm{~d} \mu \leq\left(\int_{A(k)}(f-k)^{p} \mathrm{~d} \mu\right)^{\frac{q}{p}}|A(k)|^{1-\frac{q}{p}} \\
& \leq\left(k^{-c} E_{c}\right)^{1-\frac{q}{p}}\left(\int_{A(k)}(f-k)^{p} \mathrm{~d} \mu\right)^{\frac{q}{p}} .
\end{aligned}
$$

Notice that by our assumption (3.1) and from the choice (3.6) we infer

$$
|A(k)| \leq k^{-c} E_{c}=E\left(2^{q}+2\right)^{\frac{c}{q-c}} \leq \vartheta_{0}\left(2^{q}+2\right)^{\frac{c}{q-c}} V(R) \leq \frac{1}{2} V(R),
$$

if $\vartheta_{0}$ is selected suitably. Therefore we may appeal to the Faber-Krahn inequality (1.13) to infer

$$
\int_{A(k)}(f-k)^{p} \mathrm{~d} \mu \leq \Lambda_{p}(|A(k)|, R)^{-1} \int_{A(k)}|\nabla f|^{p} \mathrm{~d} \mu,
$$

and from (3.6)-(3.9) we get, also by exploiting again $|A(k)| \leq k^{-\beta} E_{\beta}$,

$$
E_{q} \leq \gamma E_{c}^{\frac{p-q+N^{-1} q p}{p-c+N^{-1} c p}}\left(\omega^{p}(E, R) \int_{B_{R}}|\nabla f|^{p} \mathrm{~d} \mu\right)^{\frac{q-c}{p-c+N^{-1} c p}}
$$

The inequality (3.2) follows elementarily.

\section{Proof of Theorem 1.11 .}

Let us use in the following the notation

$$
\begin{gathered}
G_{1}(t, R)=\sup _{0<\tau<t} \int_{B_{R}\left(x_{0}\right)} u(x, \tau) \mathrm{d} \mu, \\
\mathcal{G}(t, R)=\max \left\{t^{-\frac{N}{\beta}} G_{1}(t, R)^{\frac{p}{\beta}}, t^{-\frac{1}{2 p+m-3}}\left(G_{1}(t, R) F(R)\right)^{\frac{p}{2 p+m-3}}\right\} .
\end{gathered}
$$

Proposition 4.1. Let $u$ be a uniformly continuous solution to (1.1)-(1.2) in $M \times$ $(0, T)$, and let $t>0$ be such that

$$
\frac{\tau}{R^{p}} u(x, \tau)^{p+m-3} \leq 1, \quad 0<\tau<t, x \in B_{(1+\sigma) R}\left(x_{0}\right),
$$

where $R \geq 1,0<\sigma<1,0<t<T$ are given. Then

$$
u(x, \tau) \leq \gamma \mathcal{G}(t,(1+\sigma) R), \quad t / 2<\tau<t, x \in B_{R}\left(x_{0}\right) .
$$

Proof. Let for all $n \geq 0$ and $a_{1}>a_{2}>0$ to be selected

$$
\begin{gathered}
r_{n}=R\left(1+\sigma\left(1-2^{-n}\right)\right), \quad r_{n}^{\prime}=\left(r_{n}+r_{n+1}\right) / 2, \quad t_{n}=t\left(1-\sigma\left(1-2^{-n}\right)\right) / 2, \\
t_{n}^{\prime}=\left(t_{n}+t_{n+1}\right) / 2, \quad r_{\infty}=R(1+\sigma), \quad t_{\infty}=t(1-\sigma) / 2, \quad k_{n}=a_{2}+\left(a_{1}-a_{2}\right) 2^{-n} .
\end{gathered}
$$


Then from Lemma 2.1 with $R_{1}=r_{n}, R_{2}=r_{n+1}$, and using also (4.1) we get for a suitable $b>1$ and for $\vartheta>0$ as in Lemma 2.1

$$
\begin{aligned}
& \sup _{t_{n}<\tau<t} \int_{B_{r_{n}}}\left(u-k_{n}\right)_{+}^{\vartheta+1} \mathrm{~d} \mu+\iint_{Q_{n}^{\prime}} \mid \nabla(u\left.-k_{n}\right)\left._{+}^{\frac{p+m+\vartheta-2}{p}}\right|^{p} \mathrm{~d} \mu \mathrm{d} \tau \\
& \leq C \frac{b^{n}}{\sigma^{p}} t^{-1} \iint_{Q_{n+1}}\left(u-k_{n+1}\right)_{+}^{\vartheta+1} \mathrm{~d} \mu \mathrm{d} \tau,
\end{aligned}
$$

where we set $Q_{n}=B_{r_{n}} \times\left(t_{n}, t\right), Q_{n}^{\prime}=B_{r_{n}^{\prime}} \times\left(t_{n}^{\prime}, t\right)$. We denote in this proof by $C$ a constant depending also on the ratio $a_{1} /\left(a_{1}-a_{2}\right)$. Let $\eta_{n}$ be a smooth cutoff function such that $\eta_{n}=1$ in $Q_{n}$ and $\eta_{n}=0$ outside of $Q_{n}^{\prime},\left|\nabla \eta_{n}\right| \leq \gamma 2^{n} / R$, $0 \leq \eta_{n t} \leq \gamma 2^{n} / t$. Define $v_{n}=\left(u-k_{n}\right)_{+}^{p+m+\vartheta-2 / p} \eta_{n}$. Then from (4.3) one gets

$$
\sup _{t_{n}<\tau<t} \int_{B_{r_{n}}} v_{n}^{\ell} \mathrm{d} \mu+\iint_{Q_{n}}\left|\nabla v_{n}\right|^{p} \mathrm{~d} \mu \mathrm{d} \tau \leq C b^{n} t^{-1} \iint_{Q_{n+1}} v_{n+1}^{\ell} \mathrm{d} \mu \mathrm{d} \tau,
$$

where $\ell=(1+\vartheta) p /(p+m+\vartheta-2)$. Next we use (3.2) as follows. First, we remark that for all $\tau, n$,

$$
\left|\operatorname{supp} v_{n}(\tau)\right| \leq\left|B_{2 R}\right| \leq \vartheta_{0} V(\gamma R)
$$

for a suitable $\gamma>1$, owing to (1.14). Set

$$
\begin{gathered}
A(s, h, \tau)=\{x: u(x, \tau)>h\} \cap B_{s}, \\
A_{n}(\tau)=A\left(r_{n}, k_{n}, \tau\right), \quad A_{\infty}(\tau)=A\left((1+\sigma) R, a_{2}, \tau\right) .
\end{gathered}
$$

Thus we may apply (3.2) in $B_{\gamma R}$, and obtain, again from (1.14) and (3.3),

$$
\begin{aligned}
\int_{B_{r_{n+1}}} v_{n+1}^{\ell} \mathrm{d} \mu \mathrm{d} \tau \leq \gamma\left|A_{n+1}(\tau)\right|^{1-\frac{\ell}{p}}+\frac{\ell}{N} & \\
& \times \omega\left(\left|A_{n+1}(\tau)\right|, R\right)^{\ell}\left(\int_{B_{r_{n+1}}}\left|\nabla v_{n+1}\right|^{p} \mathrm{~d} \mu\right)^{\frac{\ell}{p}} .
\end{aligned}
$$

Hence by means of Young's inequality and integrating in time we find

$$
\begin{aligned}
& \iint_{Q_{n+1}} v_{n+1}^{\ell} \mathrm{d} \mu \mathrm{d} \tau \leq \gamma \delta^{\frac{p}{\ell}} \iint_{Q_{n+1}}\left|\nabla v_{n+1}\right|^{p} \mathrm{~d} \mu \mathrm{d} \tau \\
& \quad+\gamma \delta^{-\frac{p}{p-\ell}} \int_{t_{n+1}}^{t}\left[\left|A_{n+1}(\tau)\right|^{1+\frac{p \ell}{N(p-\ell)}} \omega\left(\left|A_{n+1}(\tau)\right|, R\right)^{\frac{p \ell}{p-\ell}}\right] \mathrm{d} \tau \\
& \quad \leq \gamma \delta^{\frac{p}{\ell}} \iint_{Q_{n+1}}\left|\nabla v_{n+1}\right|^{p} \mathrm{~d} \mu \mathrm{d} \tau \\
& \quad+\gamma \delta^{-\frac{p}{p-\ell} t} \sup _{t_{n+1}<\tau<t}\left[\left|A_{n+1}(\tau)\right|^{1+\frac{p \ell}{N(p-\ell)}} \omega\left(\left|A_{n+1}(\tau)\right|, R\right)^{\frac{p \ell}{p-\ell}}\right] .
\end{aligned}
$$


It is immediately seen that the inequality above implies for any fixed $\varepsilon>0$, for a suitable choice of $\delta>0$, that

$$
\begin{aligned}
& C b^{n} t^{-1} \iint_{Q_{n+1}} v_{n+1}^{\ell} \mathrm{d} \mu \mathrm{d} \tau \leq \varepsilon \iint_{Q_{n+1}}\left|\nabla v_{n+1}\right|^{p} \mathrm{~d} \mu \mathrm{d} \tau \\
& \quad+C b_{1}^{n} \varepsilon^{-\frac{\ell}{p-\ell}} t^{-\frac{\ell}{p-\ell}} \sup _{t_{n+1}<\tau<t}\left[\left|A_{n+1}(\tau)\right|^{1+\frac{p \ell}{N(p-\ell)}} \omega\left(\left|A_{n+1}(\tau)\right|, R\right)^{\frac{p \ell}{p-\ell}}\right]
\end{aligned}
$$

Thus (4.4) and (4.5) give us

$$
\begin{aligned}
\sup _{t_{n}<\tau<t} \int_{B_{r_{n}}} v_{n}^{\ell} \mathrm{d} \mu & +\iint_{Q_{n}}\left|\nabla v_{n}\right|^{p} \mathrm{~d} \mu \mathrm{d} \tau \leq \varepsilon \iint_{Q_{n+1}}\left|\nabla v_{n+1}\right|^{p} \mathrm{~d} \mu \mathrm{d} \tau \\
& +C b_{1}^{n} \varepsilon^{-\frac{\ell}{p-\ell}} t^{-\frac{\ell}{p-\ell}} \sup _{t_{\infty}<\tau<t}\left[\left|A_{\infty}(\tau)\right|^{1+\frac{p \ell}{N(p-\ell)}} \omega\left(\left|A_{\infty}(\tau)\right|, R\right)^{\frac{p \ell}{p-\ell}}\right] .
\end{aligned}
$$

On iterating this inequality for $n \rightarrow \infty$, we find that actually

$$
\begin{aligned}
& \sup _{t / 2<\tau<t} \int_{B_{R}}\left(u-a_{1}\right)_{+}^{1+\vartheta} \mathrm{d} \mu \leq C(\sigma) t^{-\frac{\ell}{p-\ell} \times} \\
& \sup _{t(1-\sigma) / 2<\tau<t}\left[\left|A\left((1+\sigma) R, a_{2}, \tau\right)\right|^{1+\frac{p \ell}{N(p-\ell)}} \omega\left(\left|A\left((1+\sigma) R, a_{2}, \tau\right)\right|, R\right)^{\frac{p \ell}{p-\ell}}\right] .
\end{aligned}
$$

We conclude the proof by further iterating (4.6). To this end, define

$$
\tau_{j}=\frac{t}{2} \sum_{n=0}^{j} 2^{-n-1}, \quad R_{j}=\frac{3}{2} R-\frac{1}{2} R \sum_{n=0}^{j} 2^{-n-1}, \quad h_{j}=h\left(1-2^{-j-1}\right),
$$

for $h>0$ to be chosen. Then from (4.6) one gets for $\bar{h}_{j}=\left(h_{j}+h_{j+1}\right) / 2$,

$$
\begin{aligned}
y_{j+1} & :=\sup _{\tau_{j+1}<\tau<t}\left|A\left(R_{j+1}, \tau, h_{j+1}\right)\right| \\
& \leq \gamma \gamma_{1}^{j} h^{-(1+\vartheta)} \sup _{\tau_{j+1}<\tau<t} \int_{B_{R_{j+1}}}\left(u-\bar{h}_{j}\right)^{1+\vartheta} \mathrm{d} \mu \\
& \leq \gamma \gamma_{1}^{j} h^{-(1+\vartheta)} t^{-\frac{1+\vartheta}{p+m-3}} y_{j}^{1+\frac{p(1+\vartheta)}{N(p+m-3)}} \omega\left(y_{j}, R\right)^{\frac{p(1+\vartheta)}{p+m-3}} .
\end{aligned}
$$

Using Lemma 5.6 p.65 of [25], we conclude that $y_{j} \rightarrow 0$ as $j \rightarrow \infty$ if

$$
h^{-1} t^{-\frac{1}{p+m-3}} y_{0}^{\frac{p}{N(p+m-3)}} \omega\left(y_{0}, R\right)^{\frac{p}{p+m-3}} \leq \gamma_{0} .
$$

Taking into account that

we see that (4.7) holds if

$$
y_{0} \leq \gamma h^{-1} G_{1}(t, 2 R)
$$

$$
\gamma h^{-1} t^{-\frac{1}{p+m-3}}\left(h^{-1} G_{1}(t, 2 R)\right)^{\frac{p}{N(p+m-3)}} \omega\left(h^{-1} G_{1}(t, 2 R), R\right)^{\frac{p}{p+m-3}}=\gamma_{0},
$$

whence we easily arrive at (4.2).

Proposition 4.2. Under the assumptions of Proposition 4.1 we have

$$
\int_{0}^{t} \int_{B_{R}}|\nabla u|^{\lambda} u^{\theta} \mathrm{d} \mu \mathrm{d} \tau \leq \gamma G_{1}(t, 2 R) t^{1-\frac{\lambda}{p}} \mathcal{G}(t, 2 R)^{s},
$$


for $0<\lambda<p, s=\lambda(3-m) / p+\theta-1>0, \theta<m-2+(p-\lambda)\left(1+N^{-1}\right)$.

The proof of Proposition 4.2 relies on Proposition 4.1, following closely the proof in the Euclidean case, see [1], [4], therefore we omit it.

Proof of Theorem 1.11. Define

$$
t_{1}=\sup \left\{t>0: \sup _{0<\tau<t} \sup _{R \geq \bar{R}} \frac{\tau}{R^{p}}\|u(\tau)\|_{\infty, B_{4 R}}^{p+m-3} \leq 1\right\} .
$$

Multiply both sides of (1.1) by a smooth cutoff function $\zeta(x)$ such that $\zeta=1$ in $B_{R}$, $\zeta=0$ outside of $B_{2 R}$ and $|\nabla \zeta| \leq \gamma / R, R \geq \bar{R}$. On applying (4.8) with $\lambda=p-1$, $\theta=m-1$ (which is admissible owing to $p+m-3>0$ ), we have for $t<t_{1}$

$$
\begin{aligned}
\int_{B_{R}} u(x, t) \mathrm{d} \mu \leq \int_{B_{2 R}} u_{0} \mathrm{~d} \mu+\frac{\gamma}{R} \int_{0}^{t} & \int_{B_{2 R}}|\nabla u|^{p-1} u^{m-1} \mathrm{~d} \mu \mathrm{d} \tau \\
\leq & \int_{B_{2 R}} u_{0} \mathrm{~d} \mu+\frac{\gamma}{R} G_{1}(t, 4 R) t^{\frac{1}{p}} \mathcal{G}(t, 4 R)^{\frac{p+m-3}{p}} .
\end{aligned}
$$

Dividing this inequality by $V(R) R^{\frac{p}{p+m-3}}$, we obtain, also by means of the doubling property (1.14),

$$
\begin{aligned}
& R^{-\frac{p}{p+m-3}} V(R)^{-1} \int_{B_{R}} u(x, t) \mathrm{d} \mu \leq \gamma R^{-\frac{p}{p+m-3}} V(R)^{-1} \int_{B_{2 R}} u_{0} \mathrm{~d} \mu \\
& +\gamma\left[\sup _{R \geq \bar{R}}(4 R)^{-\frac{p}{p+m-3}} V(4 R)^{-1} \int_{B_{4 R}} u(x, t) \mathrm{d} \mu\right] \frac{t^{\frac{1}{p}}}{R} \mathcal{G}(t, 4 R)^{\frac{p+m-3}{p}} .
\end{aligned}
$$

Define also for a $\delta>0$ to be chosen

$$
t_{2}=\sup \left\{t>0: \sup _{R \geq \bar{R}} \frac{t^{\frac{1}{p}}}{R} \mathcal{G}(t, 8 R)^{\frac{p+m-3}{p}} \leq \delta\right\} .
$$

Indeed $t_{2}>0$ since $t \mapsto \mathcal{G}(t, R)^{p+m-3}$ is increasing. Let $t_{0}=\min \left\{t_{1}, t_{2}\right\}$. Then for $t<t_{0}$ we get from (4.9)

$$
\begin{aligned}
& \sup _{R \geq \bar{R}} R^{-\frac{p}{p+m-3}} V(R)^{-1} \int_{B_{R}} u(x, t) \mathrm{d} \mu \leq \gamma\left\|u_{0}\right\|_{\bar{R}} \\
& +\gamma \delta\left[\sup _{0<\tau<t} \sup _{R \geq \bar{R}}(4 R)^{-\frac{p}{p+m-3}} V(4 R)^{-1} \int_{B_{4 R}} u(x, t) \mathrm{d} \mu\right] .
\end{aligned}
$$

Hence for a sufficiently small $\delta$ we obtain

$$
\|u(t)\|_{\bar{R}} \leq \gamma\left\|u_{0}\right\|_{\bar{R}} \quad 0<t<t_{0} .
$$

We claim that $t_{0}=t_{2}$. Indeed, for $R \geq \bar{R}, t<t_{0}$,

$$
\frac{t}{R^{p}}\|u(t)\|_{\infty, B_{4 R}}^{p+m-3} \leq \gamma \frac{t}{R^{p}} \mathcal{G}(t, 8 R)^{p+m-3} \leq \gamma \delta^{p}<\frac{1}{2},
$$


for a suitable selection of $\delta$. Next we bound below $t_{0}=t_{2}$. We have

$$
\begin{aligned}
& \sup _{R \geq \bar{R}} \frac{t^{\frac{1}{p}}}{R} \mathcal{G}(t, 8 R)^{\frac{p+m-3}{p}} \leq \gamma \sup _{R \geq \bar{R}} \max \{ \\
& t^{\frac{1}{N(p+m-3)+p}} \sup _{0<\tau<t}\left(\frac{1}{R^{N+\frac{p}{p+m-3}}} \int_{B_{8 R}} u(x, \tau) \mathrm{d} \mu\right)^{\frac{p+m-3}{N(p+m-3)+p}} \\
& \left.t^{\frac{1}{2 p+m-3}} \sup _{0<\tau<t}\left(\frac{1}{V(R) R^{\frac{p}{p+m-3}}} \int_{B_{8 R}} u(x, \tau) \mathrm{d} \mu\right)^{\frac{p+m-3}{2 p+m-3}}\right\} .
\end{aligned}
$$

Thus, invoking also (4.10), as well as the monotonic character of $V(R) / R$, which follows from H.2, and implies $V(R) \leq \gamma R^{N}, R>1$, we arrive at

$$
\begin{aligned}
& \delta=\sup _{R \geq \bar{R}} \frac{t_{0}^{\frac{1}{p}}}{R} \mathcal{G}\left(t_{0},\right.4 R)^{\frac{p+m-3}{p}} \\
& \leq \gamma \max \left\{t_{0}^{\overline{N(p+m-3)+p}}\left\|u_{0}\right\| \frac{1}{\frac{p+m-3}{(p+m-3)+p}}, t_{0}^{\frac{1}{2 p+m-3}}\left\|u_{0}\right\| \frac{p+m-3}{R}{ }^{\frac{p+m-3}{R}}\right\} .
\end{aligned}
$$

On defining $T_{0}=t_{0}$ we have indeed $T_{0}=\gamma\left\|u_{0}\right\| \frac{3-m-p}{R}$ and (1.16). Finally, (4.2), (4.10) and (4.11) yield

$$
\begin{aligned}
&\|u(t)\|_{\infty, B_{R}} \leq \gamma R^{\frac{p}{p+m-3}} \times \\
& \max \left\{t^{-\frac{N}{N(p+m-3)+p}}\left\|u_{0}\right\| \frac{\frac{p}{R(p+m-3)+p}}{N}, t^{-\frac{1}{2 p+m-3}}\left\|u_{0}\right\| \frac{p}{\frac{p}{R}+m-3}\right\},
\end{aligned}
$$

whence (1.17).

In order to prove (1.18) we first note that if $\left\|u_{0}\right\|_{1}<\infty$, then $T_{0}$ can be chosen as large as needed by choosing a large enough $\bar{R}$ and by invoking conservation of mass. Therefore (1.18) follows from (4.2) and again by conservation of mass.

\section{Proof of Theorem 1.12 .}

Denote for the sake of brevity

$$
\begin{aligned}
& P(t)=\mathcal{Z}\left(\left\|u_{0}\right\|_{1, M}^{m+p-3} t\right), \quad\left\|u_{0}\right\|_{1, M}^{m+p-3} t \geq 1, \\
& P(t)=\mathcal{Z}(1), \quad\left\|u_{0}\right\|_{1, M}^{m+p-3} t<1 .
\end{aligned}
$$

First we use the local estimates which we already proved to obtain a bound for the moment of $u(t)$ in terms of the norm $\|u(t)\|_{\infty}$.

Lemma 5.1. Assume $I_{0}<\infty$. Then for all $t>0$

$$
I(t) \leq \gamma I_{0}+\gamma F(H(t))\left\|u_{0}\right\|_{1},
$$

where for all $\vartheta \in(0,1 / p)$

$$
H(t)=t^{\frac{1}{p}-\vartheta}\left(\int_{0}^{t} \tau^{\frac{\vartheta p}{p-1}-1}\|u(\tau)\|_{\infty^{\frac{p+m-3}{p-1}}} \mathrm{~d} \tau\right)^{\frac{p-1}{p}} .
$$


Proof. Let us fix $R \geq 1$. We denote here $d(x)=\operatorname{dist}\left(x, x_{0}\right)$. Let $\eta=\zeta^{p}(d(x)) F(d(x))$ where $\zeta(s), s \geq 0$ is a smooth function such that $\zeta(s)=1$ for $s \geq 2 R, \zeta(s)=0$ for $0 \leq s \leq R, 0 \leq \zeta^{\prime} \leq \gamma / R, 0 \leq \zeta \leq 1$. We also remark that owing to (1.15)

$$
|\nabla F(d(x))| \leq F^{\prime}(d(x)) \leq \frac{1}{V(d(x))}, \quad d(x)>1 .
$$

Multiplying against $\eta$ both sides of (1.1) and integrating by parts, we get

$$
\begin{aligned}
& \int_{M \backslash B_{R}} \zeta^{p} F u(x, t) \mathrm{d} \mu \leq I_{0}+\int_{0}^{t} \int_{M \backslash B_{R}} u^{m-1}|\nabla u|^{p-1} \frac{1}{V(d(x))} \zeta^{p} \mathrm{~d} \mu \mathrm{d} \tau \\
& \quad+p \int_{0}^{t} \int_{B_{2 R} \backslash B_{R}} u^{m-1}|\nabla u|^{p-1} F(d(x))|\nabla \zeta| \zeta^{p-1} \mathrm{~d} \mu \mathrm{d} \tau \\
& \leq I_{0}+\frac{\gamma}{V(R)} \int_{0}^{t} \int_{M} u^{m-1}|\nabla u|^{p-1} \mathrm{~d} \mu \mathrm{d} \tau .
\end{aligned}
$$

Next we estimate the second term on the right-hand side of (5.2) as follows. Let $\bar{R} \geq 1$ and $\psi$ be a standard cutoff function in $B_{2}$, such that $\psi=1$ in $B_{\bar{R}}$. Then by Hölder's inequality one gets

$$
J:=\int_{0}^{t} \int_{B_{2 \bar{R}}} \psi^{p} u^{m-1}|\nabla u|^{p-1} \mathrm{~d} \mu \mathrm{d} \tau \leq I_{1}^{\frac{p-1}{p}} I_{2}^{\frac{1}{p}},
$$

where

$$
\begin{aligned}
& I_{1}=\int_{0}^{t} \int_{B_{2 \bar{R}}} \tau^{\frac{\vartheta p}{p-1}} \psi^{p} u^{\frac{(m-1) p}{p-1}-\frac{1}{p-1}}|\nabla u|^{p} \mathrm{~d} \mu \mathrm{d} \tau, \\
& I_{2}=\int_{0}^{t} \int_{B_{2 \bar{R}}} \tau^{-\vartheta p} \psi^{p} u \mathrm{~d} \mu \mathrm{d} \tau \leq \gamma t^{1-\vartheta p}\left\|u_{0}\right\|_{1} .
\end{aligned}
$$

To estimate $I_{1}$ we choose in the equation as a test function $\tau^{\frac{\vartheta p}{p-1}} u^{\frac{p+m-3}{p-1}} \psi^{p}$. Then we get by integration by parts and Young's inequality

$$
\begin{aligned}
I_{1} \leq \frac{\gamma}{\bar{R}^{p}} \int_{0}^{t} \int_{B_{2 \bar{R}}} \tau^{\frac{\vartheta p}{p-1}} u^{p+m-2+\frac{p+m-3}{p-1}} & \mathrm{~d} \mu \mathrm{d} \tau \\
& +\gamma \int_{0}^{t} \int_{B_{2 \bar{R}}} \tau^{\frac{\vartheta p}{p-1}-1} u^{1+\frac{p+m-3}{p-1}} \mathrm{~d} \mu \mathrm{d} \tau=I_{3}+I_{4} .
\end{aligned}
$$

Since we are going to let $\bar{R} \rightarrow \infty$ we may assume, for any fixed $\tau>0$, that in (1.18) [written for $R=2 \bar{R}, t=\tau$ ] the second term on the right hand side is the greater one. Assume $\bar{R} \geq \bar{\gamma} P(\tau)$, where $\bar{\gamma}$ is such that (1.18) implies

$$
\tau(2 \bar{R})^{-p}\|u(\tau)\|_{\infty, B_{2} \bar{R}}^{p+m-3} \leq 1
$$


Then the integrand in $I_{3}$ is majorised by the one in $I_{4}$ (at time $\tau$ ). On recalling that we are working with smooth approximating solutions, by invoking Fatou's lemma it is easily seen that in the limit $\bar{R} \rightarrow \infty$ equation (5.3) gives

$$
\begin{aligned}
\int_{0}^{t} \int_{M} u^{m-1}|\nabla u|^{p-1} \mathrm{~d} \mu \mathrm{d} \tau & =\lim _{\bar{R} \rightarrow \infty} J \leq \\
\gamma\left\|u_{0}\right\|_{1} t^{\frac{1}{p}-\vartheta} & \left(\int_{0}^{t} \tau^{\vartheta \frac{p}{p-1}-1}\|u(\tau)\|_{\infty^{\frac{p+m-3}{p-1}}} \mathrm{~d} \tau\right)^{\frac{p-1}{p}}=\gamma\left\|u_{0}\right\|_{1} H(t) .
\end{aligned}
$$

Therefore we have, by using also (5.2),

$$
\begin{aligned}
I(t)=\int_{B_{R}} F(d(x)) u(x, t) \mathrm{d} \mu+\int_{M \backslash B_{R}} F( & d(x)) u(x, t) \mathrm{d} \mu \\
& \leq F(R)\left\|u_{0}\right\|_{1}+\gamma I_{0}+\frac{\gamma}{V(R)} H(t)\left\|u_{0}\right\|_{1} .
\end{aligned}
$$

If $H(t) \geq 1$ we may select above $R=H(t)$ to obtain (5.1). If $H(t)<1$ we choose $R=1$ to get the same result.

Finally we absorb the $L^{\infty}$ norm appearing in the right hand side of (5.1) with the help of two technical lemmas proven in [2], which we reproduce in our notation for the reader's convenience.

Lemma 5.2. [2] If $A, B>0, \Gamma>1$ satisfy

$$
B \leq A F(\Gamma)^{p /(2 p+m-3)},
$$

then for all $\varepsilon>0$ there exists $c_{\varepsilon}>1$ such that for all $\sigma>0$

$$
\sigma B^{(p+m-3) / p} \leq \varepsilon \Gamma+c_{\varepsilon} \mathcal{Z}_{1}\left(\left(\sigma A^{\frac{p+m-3}{p}}\right)^{2 p+m-3}\right),
$$

where $\mathcal{Z}_{1}(s)=\mathcal{Z}(\max (s, 1)), s>0$.

Lemma 5.3. [2] Let $g \in C((0, \infty)), g \geq 0$ satisfy

$$
g(t) \leq U(t)+\varepsilon t^{-\sigma}\left(\int_{0}^{t} \tau^{\sigma q-1} g(\tau)^{q} \mathrm{~d} \tau\right)^{1 / q}, \quad t>0,
$$

where $\varepsilon, \sigma, q>0$ are given, and $U \in C((0, \infty)), U>0$. Assume also that, for $t>0$ and a given $g_{0} \in C((0, \infty))$, we have $g(t) \leq g_{0}(t)$, and that for a given $0<\delta<\sigma$, $t^{\delta} g_{0}(t), t^{\delta} U(t)$ are non decreasing in $t>0$. Then, if $\varepsilon<\eta=(\sigma q-\delta q)^{1 / q}$, we have

$$
g(t) \leq(1-\varepsilon / \eta)^{-1} U(t), \quad t>0 .
$$

Proof of Theorem 1.12. Proceeding as in the proof of Proposition 4.1 one can show that

$$
\begin{aligned}
\|u(t)\|_{\infty, A_{R}} \leq \gamma \max & \left\{t^{-\frac{N}{\beta}}\left(\sup _{0<\tau<t} \int_{A_{R}^{\prime}} u(x, \tau) \mathrm{d} \mu\right)^{\frac{p}{\beta}},\right. \\
& \left.t^{-\frac{1}{2 p+m-3}}\left(\sup _{0<\tau<t} \int_{A_{R}^{\prime}} u(x, \tau) \mathrm{d} \mu\right)^{\frac{p}{2 p+m-3}} F(R)^{\frac{p}{2 p+m-3}}\right\},
\end{aligned}
$$


for all $R \geq \gamma P(t)$ where $A_{R}=B_{2 R} \backslash B_{R}$ and $A_{R}^{\prime}=B_{4 R} \backslash B_{R / 2}$. Then the last term in (5.4) can be bounded in terms of $I(t)$, which in turn can be estimated as in (5.1). Thus we have

$$
\begin{aligned}
\|u(t)\|_{\infty} \leq & \\
& \tilde{\gamma} \max \left\{\mathcal{U}(t), t^{-1 /(2 p+m-3)}\left\|u_{0}\right\|_{1}^{p /(2 p+m-3)} F(H(t))^{p /(2 p+m-3)}\right\} .
\end{aligned}
$$

For the sake of logical clarity, let us fix from now $\varepsilon, \vartheta, \delta$ so that

$$
\varepsilon=\frac{1}{4}\left(\frac{(\vartheta-\delta) p}{p-1}\right)^{\frac{p-1}{p}}, \quad \frac{N(p+m-3)}{\beta p}<\delta<\vartheta<\frac{1}{p} .
$$

Let $C=\tilde{c}_{\varepsilon} / \varepsilon>1$, where for $c_{\varepsilon}$ as in Lemma 5.2, and for $\tilde{\gamma}$ as in (5.5),

$$
\tilde{c}_{\varepsilon}=\gamma_{1} c_{\varepsilon}, \quad \gamma_{1}=\tilde{\gamma}^{(2 p+m-3)(p+m-3) / p}>1 .
$$

If $H(t)>C P(t)$, so that $H(t)>1$, we may apply Lemma 5.2 , which is a functional version of Young's inequality, to get from (5.5)

$$
t^{1 / p}\|u(t)\|_{\infty}^{(p+m-3) / p} \leq \max \left\{\varepsilon H(t)+\tilde{c}_{\varepsilon} P(t), \gamma \mathcal{U}(t)^{(p+m-3) / p} t^{1 / p}\right\} .
$$

Indeed, we select to this end in Lemma 5.2

$$
\Gamma=H(t) \quad B=\|u(t)\|_{\infty}, \quad \sigma=t^{1 / p}, A=\tilde{\gamma} t^{-1 /(2 p+m-3)}\left\|u_{0}\right\|_{1}^{p /(2 p+m-3)} ;
$$

here we also use the fact

$$
\mathcal{Z}\left(\gamma_{1} t\left\|u_{0}\right\|_{1}^{p+m-3}\right) \leq \gamma_{1} \mathcal{Z}\left(t\left\|u_{0}\right\|_{1}^{p+m-3}\right),
$$

which follows from the definition of $\mathcal{Z}$. Actually (5.6) implies

$$
\|u(t)\|_{\infty}^{(p+m-3) / p} \leq 2 \varepsilon t^{-1 / p} H(t)+\gamma \mathcal{U}(t)^{(p+m-3) / p},
$$

when we use our assumption $H(t)>C P(t)$ again. However, (5.7) is in force even if $H(t) \leq C P(t)$, as it follows from (5.1), (5.5), and the definitions of $\mathcal{U}(t)$ and $P(t)$, perhaps by an inessential change of the constant $\gamma$ appearing in (5.7). Thus (5.7) is in force for all $t>0$.

Notice that (5.7) is in practice an integral inequality; if we knew the monotonicity in $t$ of $t^{\delta}\|u(t)\|_{\infty}$, for a suitable $\delta>0$, the statement would follow readily. In the general case, our choice of $\varepsilon$ enables us to apply Lemma 5.3 to circumvent the technical difficulty posed by the lack of monotonicity and prove (1.19). Indeed, we may select in Lemma 5.3

$$
\begin{gathered}
g(t)=\|u(t)\|_{\infty}^{(p+m-3) / p}, \quad U(t)=\gamma \mathcal{U}(t)^{(p+m-3) / p}, \\
\sigma=\vartheta, \quad q=p /(p-1), \quad g_{0}(t)=\left\|u_{0}\right\|_{\infty},
\end{gathered}
$$

and $\varepsilon, \delta$ and $\vartheta$ as above.

\section{REFERENCES}

[1] D. Andreucci. Degenerate parabolic equations with initial data measures. Transactions American Mathematical Society, 349:3911-3923, 1997. American Mathematical Society.

[2] D. Andreucci and A. F. Tedeev. Optimal bounds and blow up phenomena for parabolic problems in narrowing domains. Proceedings Royal Soc. Edinburgh, 128A:1163-1180, 1998. RSE Scotland Foundation, Edinburgh (UK).

[3] D. Andreucci and A. F. Tedeev. A Fujita type result for a degenerate Neumann problem in domains with non compact boundary. J. Math. Analysis and Appl., 231:543-567, 1999. Elsevier. 
[4] D. Andreucci and A. F. Tedeev. Sharp estimates and finite speed of propagation for a Neumann problem in domains narrowing at infinity. Advances Diff. Eqs., 5:833-860, 2000. Khayyam Publ., Athens Ohio (U.S.A.).

[5] D. Andreucci and A. F. Tedeev. Universal bounds at the blow-up time for nonlinear parabolic equations. Advances in Differential Equations, 10:89-120, 2005. Khayyam Publ., Athens Ohio (U.S.A.).

[6] M. Bonforte and G. Grillo. Super and ultracontractive bounds for doubly nonlinear evolution equations. Rev. Mat. Iberoam., 22(1):111-129, 2006.

[7] M. Bonforte and G. Grillo. Singular evolution on manifolds, their smoothing properties, and Sobolev inequalities. Discrete Contin. Dyn. Syst., (Dynamical Systems and Differential Equations. Proceedings of the 6th AIMS International Conference, suppl.):130-137, 2007.

[8] M. Bonforte, G. Grillo, and J. L. Vazquez. Fast diffusion flow on manifolds of nonpositive curvature. J. Evol. Equ., 8(1):99-128, 2008.

[9] I. Chavel. Eigenvalues in Riemannian geometry, volume 115 of Pure and Applied Mathematics. Academic Press Inc., Orlando, FL, 1984. Including a chapter by Burton Randol, With an appendix by Jozef Dodziuk.

[10] I. Chavel and E. A. Feldman. Isoperimetric inequalities on curved surfaces. Adv. in Math., 37(2):83-98, 1980.

[11] I. Chavel and E. A. Feldman. Modified isoperimetric constants, and large time heat diffusion in Riemannian manifolds. Duke Math. J., 64(3):473-499, 1991.

[12] F. Cipriani and G. Grillo. Uniform bounds for solutions to quasilinear parabolic equations. J. Differential Equations, 177(1):209-234, 2001.

[13] T. Coulhon. Ultracontractivity and Nash type inequalities. J. Funct. Anal., 141(2):510-539, 1996.

[14] T. Coulhon, A. Grigor'yan, and D. Levin. On isoperimetric profiles of product spaces. Comm. Anal. Geom., 11(1):85-120, 2003.

[15] S. A. J. Dekkers. Finite propagation speed for solutions of the parabolic $p$-Laplace equation on manifolds. Comm. Anal. Geom., 13(4):741-768, 2005.

[16] S. A. J. Dekkers. A comparison theorem for solutions of degenerate parabolic equations on manifolds. Proc. Roy. Soc. Edinburgh Sect. A, 138(4):755-767, 2008.

[17] A. Grigor'yan. Heat kernel on a manifold with a local Harnack inequality. Comm. Anal. Geom., 2(1):111-138, 1994.

[18] A. Grigor'yan. Heat kernel upper bounds on a complete non-compact manifold. Rev. Mat. Iberoamericana, 10(2):395-452, 1994.

[19] A. Grigor'yan. Isoperimetric inequalities and capacities on Riemannian manifolds. In The Maz'ya anniversary collection, Vol. 1 (Rostock, 1998), volume 109 of Oper. Theory Adv. Appl., pages 139-153. Birkhäuser, Basel, 1999.

[20] A. Grigor'yan and L. Saloff-Coste. Heat kernel on manifolds with ends. Ann. Inst. Fourier (Grenoble), 59(5):1917-1997, 2009.

[21] G. Grillo and M. Muratori. Sharp short and long time $L^{\infty}$ bounds for solutions to porous media equations with homogeneous Neumann boundary conditions. J. Differential Equations, 254(5):2261-2288, 2013.

[22] G. Grillo, M. Muratori, and M. M. Porzio. Porous media equations with two weights: smoothing and decay properties of energy solutions via Poincaré inequalities. Discrete Contin. Dyn. Syst., 33(8):3599-3640, 2013.

[23] A. K. Gushchin. The rate of stabilization of a solution of a parabolic equation in an unbounded region. Diff. Uravn., 6:741-761, 1970. Engl. Transl. Diff. Eqns. 6, 1970, 567-581.

[24] A. K. Gushchin. On the uniform stabilization of solutions of the second mixed problem for a parabolic equation. Mat. Sbornik, 119(161), 1982. Engl. Transl. Math. USSR Sbornik 47, 1984, 439-498.

[25] O. A. Ladyzhenskaja, V. A. Solonnikov, and N. N. Ural'ceva. Linear and Quasilinear Equations of Parabolic Type, volume 23 of Translations of Mathematical Monographs. American Mathematical Society, Providence, RI, 1968.

[26] A. V. Lezhnev. On the behaviour, for large time values, of nonnegative solutions of the second mixed problem for a parabolic equation. Mat. Sbornik, 129(171), 1986. Engl. Transl. Math. USSR Sbornik 57, 1987, 195-209.

[27] S. Sakaguchi. Concavity properties of solutions to some degenerate quasilinear elliptic Dirichlet problems. Ann. Scuola Norm. Sup. Pisa Cl. Sci. (4), 14(3):403-421 (1988), 1987. 
[28] J. Xiao. The p-Faber-Krahn inequality noted. In Around the research of Vladimir Maz'ya. I, volume 11 of Int. Math. Ser. (N. Y.), pages 373-390. Springer, New York, 2010. 